Original Article

Joumal of Epilepsy Research pISSN 2233-6249 / eISSN 2233-6257

Received September 10, 2015

Accepted January 10, 2016

Corresponding author: Carl E. Stafstrom Division of Pediatric Neurology, Johns Hop kins University, 200 North Wolfe Street Baltimore, MD 21287, USA

Tel. +1-410-955-4259

Fax. +1-410-614-2297

E-mail; cstafst1@jhmi.edu

\section{Ketogenic Diet, but Not Polyunsaturated Fatty Acid Diet, Reduces Spontaneous Seizures in Juvenile Rats with Kainic Acid-induced Epilepsy}

\author{
Simone M. Dustin, Carl E. Stafstrom \\ Department of Neurology, University of Wisconsin School of Medicine and Public Health, Madison, Madison, USA
}

\begin{abstract}
Background and Purpose: The high-fat, low-carbohydrate ketogenic diet (KD) is effective in many cases of drug-resistant epilepsy, particularly in children. In the classic KD, fats consist primarily of long-chain saturated triglycerides. Polyunsaturated fatty acids (PUFAs), especially the n-3 type, decrease neuronal excitability and provide neuroprotection; pilot human studies have raised the possibility of using PUFAs to control seizures in patients.
\end{abstract}

Methods: To determine the relative roles of the KD and PUFAs in an animal model, we induced epilepsy in juvenile rats (P29-35) using intraperitoneal kainic acid (KA). KA caused status epilepticus in all rats. Two days after KA, rats were randomized to one of 4 dietary groups: Control diet; PUFA diet; KD; or KD plus PUFA. All diets were administered isocalorically at $90 \%$ of the rat recommended daily calorie requirement. Spontaneous recurrent seizures (SRS) were assessed for 3 months after diet randomization.

Results: Rats receiving the KD or KD-PUFA diet had significantly fewer SRS than those receiving the Control diet or PUFA diet. The PUFA diet did not reduce SRS compared to the Control diet.

Conclusions: In the KA epilepsy model, the KD protects against SRS occurrence but dietary enhancement with PUFA does not afford additional protection against spontaneous seizures. (2016;6:1-7)

Key words: Ketogenic diet, Polyunsaturated fatty acids, Epilepsy, Neuroprotection, Kainic acid, Animal models

\section{Introduction}

The ketogenic diet (KD) is effective in many cases of medically refractory epilepsy, especially in children, but the diet is often difficult to administer and maintain. ${ }^{1}$ Studies of KD mechanisms of action have focused on the role of ketosis, with relatively less attention paid to the roles of the type and quantity of fat. Although ketosis is necessary for the action of the KD, there is an imprecise correlation between the level of ketosis and seizure control, ${ }^{2-4}$ suggesting that additional mechanisms may be involved. Some reports correlate seizure improvement with higher fat ratios. ${ }^{3,5-7}$ In addition to fat content, fat type (e.g., degree of saturation or chain length) may play a role in altering neuronal excitability.

Polyunsaturated fatty acids (PUFAs) are dietary lipids necessary for optimal brain development and function. ${ }^{8,9}$ PUFAs, especially those of the n-3 (omega-3) class, also exert a stabilizing effect on cardiac and neuronal membrane excitability, possibly by altering sodium or calcium channel function ${ }^{10-12}$ or other mechanisms such as an effect on chloride transporters. ${ }^{13}$ The classic KD consists mostly of long-chain saturated triglycerides and has a low concentration of PUFAs, though increased PUFAs have been documented in children on the KD. ${ }^{14} n-3$ PUFAs, especially docosahexaenoic acid (DHA) and eicosapentaenoic acid (EPA), have been shown to reduce neuronal excitability in several experimental systems including animal models, hippocampal slices, and dissociated neurons in culture. ${ }^{15-17}$ Systemic administration of PUFAs raises the threshold for seizure generation in some acute models ${ }^{18-20}$ but not others. ${ }^{21,22}$ Similarly, clinical studies of PUFAs in patients with epilepsy have yielded inconsistent results several case reports or small series suggested a beneficial effect on seizure frequency ${ }^{23-25}$ but clinical trials failed to show a protective effect. $^{26-29}$

Kainic acid $(K A)$ is a glutamate analog that causes status epilepticus in rodents after systemic administration, followed by the development of spontaneous recurrent seizures over the ensuing weeks 
to months. ${ }^{30,31}$ The KD has been shown to exert an antiepileptogenic effect on P30 rats after KA-induced status epilepticus, with prevention of spontaneous recurrent seizures (SRS) when the diet was started 2 days but not 14 days after status epilepticus. ${ }^{32}$ To determine whether dietary PUFAs, alone or as a supplement to the classic KD, affect SRS frequency and epilepsy development, we studied the effects of 4 different diets using KA-induced epilepsy in juvenile rats. We tested the hypothesis that a diet enriched in PUFAs exerts an antiepileptic effect.

\section{Methods}

\section{Subjects and diets}

This study was approved by the University of Wisconsin Institutional Animal Care and Use Committee. We compared the following diets: a standard diet consisting of rat chow (Control), a rat chow diet supplemented with PUFAs (PUFA), a classic KD (KD), and a classic KD diet supplemented with PUFA (KD-PUFA) (Table 1). On P29-35, male Sprague-Dawley rats (Harlan, Madison, WI) were subjected to status epilepticus induced by KA $10 \mathrm{mg} / \mathrm{kg}$ i.p. Status epilepticus was stopped after 2 hours using diazepam (5-10 mg/kg, i.p.). Two days later, following recovery from status epilepticus, rats were randomly assigned to continue on a control diet $(n=8)$ or were switched to one of 3 experimental diets: PUFA $(n=10), \operatorname{KD}(n=10)$, or KD-PUFA ( $n=$ 10). Eight additional rats received saline i.p. (no KA), were fed the Control diet and were observed for SRS along with the rats that had undergone KA-induced status epilepticus. All diets were administered isocalorically with calories restricted to $90 \%$ of the recommended daily requirement for rats $(0.3 \mathrm{kcal} / \mathrm{g}$ body weight/day), to simulate the calorie restriction typically used in patients on the KD. ${ }^{1}$
Rats were fed daily between 15:00 and 17:00. The daily amount of food provided was calculated according to the formula: body weight (g) $\times(0.3 \mathrm{kcal} /$ body weight $(\mathrm{g})) \times$ food $(\mathrm{g}) / \mathrm{kcal} \times 0.9=$ food administered (g). ${ }^{33,34}$ Any unconsumed food was discarded at the next feeding, but this rarely occurred as rats typically consumed all of their daily allotment. Water was provided ad libitum to all groups.

PUFA-containing menhaden oil accounts for $20 \%$ of the diet content by food weight. The exact amount of PUFAs consumed in the KD-PUFA and PUFA diets depended on the amount of food ingested overall, which varied between 2.5 and 15 grams per day, depending on the age of the rat. Therefore, rats receiving the KD-PUFA or PUFA diets in this study consumed between 200 and 2,000 mg of PUFA per day. DHA, the major n-3 PUFA in the brain, comprises about 21.5\% of total PUFAs in the PUFA diet, so rats consumed approximately 43-430 mg of DHA per day. See Table 1 for further diet details.

\section{Spontaneous seizure monitoring}

Beginning about 1 week following status epilepticus and continuing for the next 3 months, rats were monitored visually for SRS by trained observers blind to diet treatment group. Each rat was observed at least twice weekly for 2-4 hours. Observations were conducted on weekday afternoons (12 p.m. - 6 p.m.) to control for diurnal variation in SRS occurrence. Each rat was observed for a minimum of 120 hours over the 3-month period. Rats from the various experimental groups were intermixed in each observation session. The number, characteristics, duration, and severity of SRS were recorded. Analysis included class III-V seizures: class III, unilateral forelimb clonus without rearing; class IV, bilateral forelimb clonus with rearing but no loss of posture; class V, bilateral forelimb clonus with rearing and loss of posture. ${ }^{35}$

Table 1. diet compositions ( $\mathrm{g} / \mathrm{kg}$ diet)

\begin{tabular}{|c|c|c|c|c|}
\hline Component & Control (\%) & PUFA (\%) & KD (\%) & KD/PUFA (\%) \\
\hline Protein & 8.36 & 8.36 & 8.2 & 8.36 \\
\hline Fat & 30.1 & $30.1^{*}$ & 75.1 & $75.1^{*}$ \\
\hline Carbohydrate & 46.8 & 46.8 & 3.2 & 3.2 \\
\hline Fiber & 4.8 & 4.8 & 4.8 & 4.8 \\
\hline Moisture & $<10$ & $<10$ & $<10$ & $<10$ \\
\hline Ash & 3 & 3 & 3 & 3 \\
\hline $\mathrm{kcal} / \mathrm{g}$ & 4.92 & 4.92 & 7.24 & 7.24 \\
\hline
\end{tabular}

Diet compositions by grams: control diet (Bio-Serve F4857, Frenchtown, NJ, USA): starch 346.1; casein 95.0; fiber 50.0; salt mix 38.0; vitamin mix 20.9; dextrose 150.0; lard 300.0. total polyunsaturated fatty acids, $33.6 \mathrm{~g} / \mathrm{kg}$ diet. PUFA diet (Bio-Serve F4858, Frenchtown, NJ, USA): starch 346.1; casein 95.0; fiber 50.0; salt mix 38.0; vitamin mix 20.9; dextrose 150.0; lard 100.0; fish oil $200.0 \mathrm{~g} / \mathrm{kg}$. Total polyunsaturated fatty acids, $79.6 \mathrm{~g} / \mathrm{kg}$ diet; DHA, approximately $17.1 \mathrm{~g} / \mathrm{kg}$ diet. Ketogenic diet (KD) (Bio-Serve F3666, Frenchtown, NJ, USA): casein 95.0; fiber 50.0; salt mix 38.0; vitamin mix 20.9; dextrose 7.6; lard 475.0; butter 199.5; corn oil 114.0. total polyunsaturated fatty acids, $122 \mathrm{~g} / \mathrm{kg}$ diet. KD/PUFA diet (Bio-Serve F4859, Frenchtown, NJ, USA): casein 95.0; fiber 50.0; salt mix 38.0; vitamin mix 20.9; dextrose 7.6; lard 275.0; butter 199.5; corn oil 114.0; fish oil $200.0 \mathrm{~g} / \mathrm{kg}$. Total polyunsaturated fatty acids, $168 \mathrm{~g} / \mathrm{kg}$ diet; dha, approximately $17.1 \mathrm{~g} / \mathrm{kg}$ diet.

"PUFA (menhaden oil), substituted for lard, comprises $20 \%$ of diet by weight. 


\section{Outcome measures and statistics}

Blood ketone ( $\beta$-hydroxybutyrate) and glucose levels were assessed at 3 months using a StatSite monitor (Stanbio Laboratory, Boerne, TX, USA). The following seizure variables were compared: latency to first SRS, number (proportion) of rats with 1 SRS, number (proportion) of rats with 5 or more SRS, SRS frequency per rat and group, mean SRS severity and mean SRS duration. Differences between dietary treatment groups were compared using Fisher's exact test for proportions or analysis of variance (ANOVA) with Bonferroni correction as appropriate, with significance considered to be $p<$ 0.05 for all tests.

\section{Results}

Status epilepticus, consisting of continuous or intermittent class IV-V seizures ${ }^{35}$ was induced in all rats and was successfully terminated at 2 hours with diazepam. At the time of status epilepticus, all rats were receiving standard rat chow (BioServe F4857). The duration and severity (seizure stage) of status epilepticus did not vary among rats treated with KA. Two days after status epilepticus, rats were randomized to the various dietary groups.

Over the following 90-100 days, rats were observed for spontaneous seizures and general health. All groups gained weight over the 3 months of dietary therapy. Weight gain was greater in the standard rat chow-fed, saline-treated rats that did not receive KA, compared to all of the KA-treated groups (one-way ANOVA with repeated measures, $F[4,31]=6.053, p<0.001$ ), which did not differ from each other. That is, there was no significant difference in weight gain between the four experimental groups (KA-con, KA-PUFA, KA-KD, KA-KD-PUFA) over the 90 days, though on day 90 , the weights of the KD-fed groups (KA-KD, KA-KD-PUFA) were less than the group of rats that were saline-injected and fed with standard rat chow (one-way ANOVA, $\mathrm{F}[4,26]=6.099, p=0.002$ ). . This data confirms previous findings that weight gain is slower on the KD or following KA treatment. ${ }^{32,36,37}$ Rats receiving the KD or KD/PUFA diets had significantly elevated serum beta-hydroxybutyrate levels compared to the other diet groups $(p=0.007)$; ketosis did not occur in the rats receiving PUFA but not KD (Fig. 1A). Serum glucose levels at 3 months did not vary significantly between dietary groups ( $p=0.227$ ) (Fig. 1B). No bleeding or bruising was observed. ${ }^{38}$

SRS are considered to represent the spectrum of focal onset seizures that can secondarily generalize. ${ }^{30}$ Selected features of SRS over the 90 days following KA status epilepticus are shown in Table 2. No
SRS were observed in control-fed rats that did not receive KA. There was no difference among KA-treated diet groups in the latency to the first SRS ( $p=0.951)$, SRS severity ( $p=0.432$ ), or SRS duration ( $p=$ 0.202 ). The number (proportion) of rats experiencing at least one spontaneous seizure was significantly lower in the KA-KD and KA-KD-PUFA diet groups compared to the KA-Control and KA-PUFA diet groups $\left(\chi^{2}[1]=6.465\right)$ (Fig. 2A, gray bars). Similarly, the number of rats experiencing 5 or more spontaneous seizures was lower in the KA-KD and KA-KD-PUFA diet groups compared to the KA-Control and KA-PUFA diet groups ( $\left.\chi^{2}[1]=4.876\right)$ (Fig. 2A, white bars). SRS frequency (SRS per hour) was significantly lower in groups receiving the KD (KA-KD, KA-KD-PUFA) than those on Control diet (KA-control) or PUFA diet (KA-PUFA) $(p=0.038)$ (Fig. 2B). However, there was no difference in SRS frequency between the KA-KD and KA-KD-PUFA groups $(p=0.626)$, suggesting that PUFA supple-

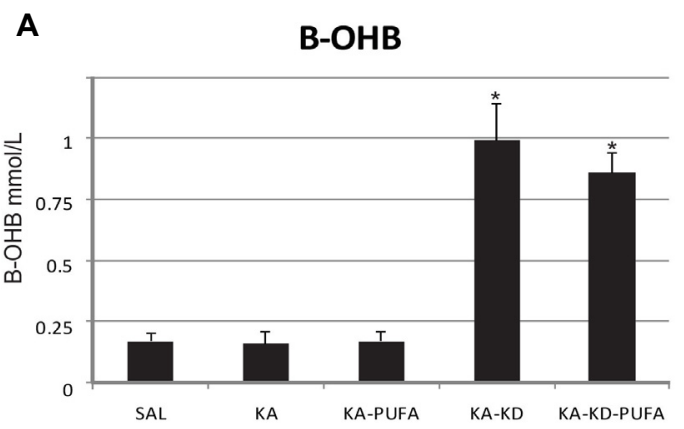

B Glucose

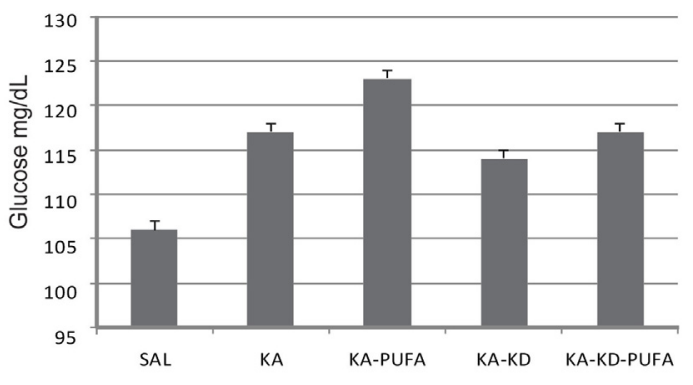

Figure 1. (A) Serum beta-hydroxybutyrate levels at 3 months as a function of dietary treatment. Rats receiving KD (KA-KD, KA-KD-PUFA) had significantly elevated serum beta-hydroxybutyrate levels compared to rats not receiving KD (SAL, KA, KA-PUFA) ( $p=0.007$; asterisks indicate significant differences compared to groups without asterisks). (B) Serum glucose levels at 3 months as a function of dietary treatment; no significant differences were found in serum glucose levels $(p=0.227)$. In all panels, data are reported as mean \pm standard error of the mean (S.E.M.). KA, kainic acid; PUFA, polyunsaturated fatty acid; KD, ketogenic diet, B-OHB, beta-hydroxybutyrate; SAL, saline. 
Table 2. Features of spontaneous recurrent seizures as a function of diet treatment in KA-treated rats

\begin{tabular}{lcccc}
\hline Diet treatment & $\mathrm{n}$ & Latency to $1_{\text {st }}$ SRS (days) & $\begin{array}{c}\text { SRS severity } \\
(\text { Racine stage })^{35}\end{array}$ & $\begin{array}{c}\text { SRS duration } \\
(\mathrm{sec})\end{array}$ \\
\hline Control & 8 & $24.0 \pm 8.1$ & $4.1 \pm 0.12$ & $39.1 \pm 4.2$ \\
PUFA & 10 & $35.0 \pm 18.8$ & $4.2 \pm 0.81$ & $34.7 \pm 2.7$ \\
KD & 10 & $32.0 \pm 13.5$ & $4.2 \pm 0.18$ & $48.7 \pm 6.1$ \\
KD + PUFA & 10 & $24.2 \pm 7.0$ & $4.3 \pm 0.17$ & $37.5 \pm 4.6$ \\
$p$ - value & & $p=0.095$ & $p=0.432$ & $p=0.202$ \\
\hline
\end{tabular}

Values given as mean \pm S.E.M. Non-significant $p$-values below each column indicate that there were no significant differences between diet groups in latency to first SRS, SRS severity score, or SRS duration.

KA, kainic acid; SRS, spontaneous recurrent seizures; sec, second; PUFA, polyunsaturated fatty acids; KD, ketogenic diet.
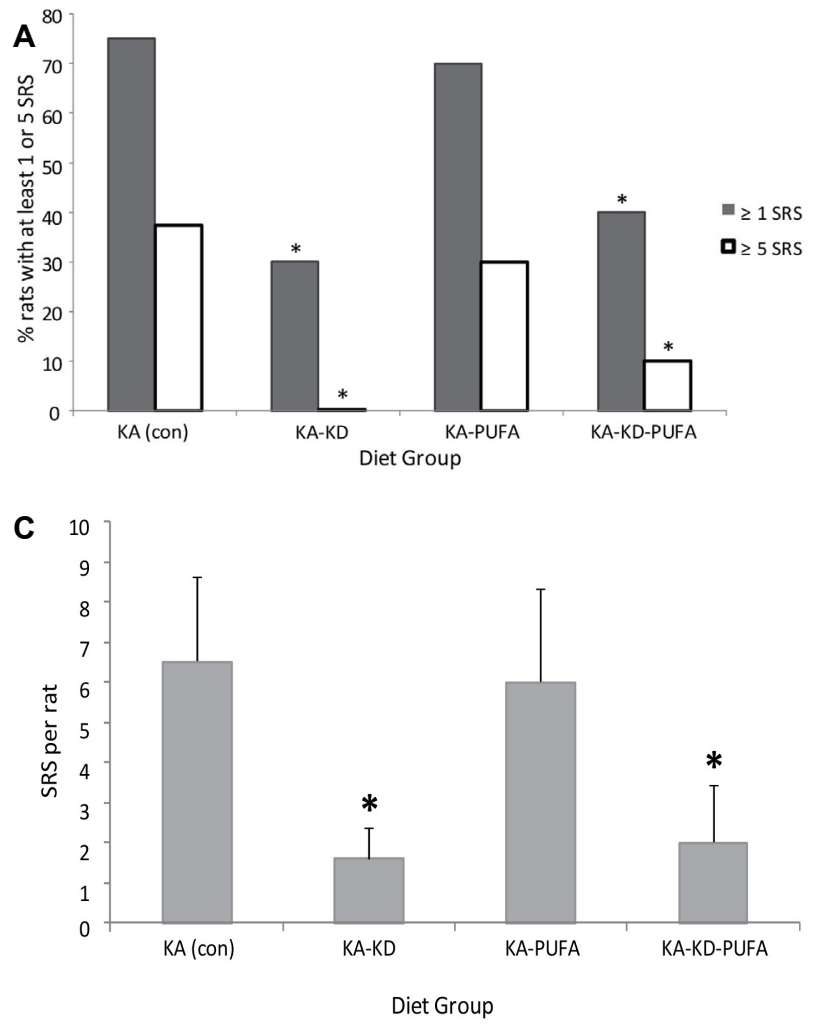

mentation did not afford greater seizure protection than the KD alone. Finally, the number of SRS per rat was significantly lower in the KA-KD and KA-KD-PUFA groups compared to the Control diet (KA-Control) or PUFA-fed rats (KA-PUFA) ( $p=0.040$ ) (Fig. 2C). SRS were observed to occur from about 1 month following status epilepticus through the end of the observation period at 3 months. There was no correlation of SRS incidence as a function of age or time of day.

\section{Discussion}

The main finding of these experiments is that the classic KD (with or without supplemental PUFA) provided protection against sponta-

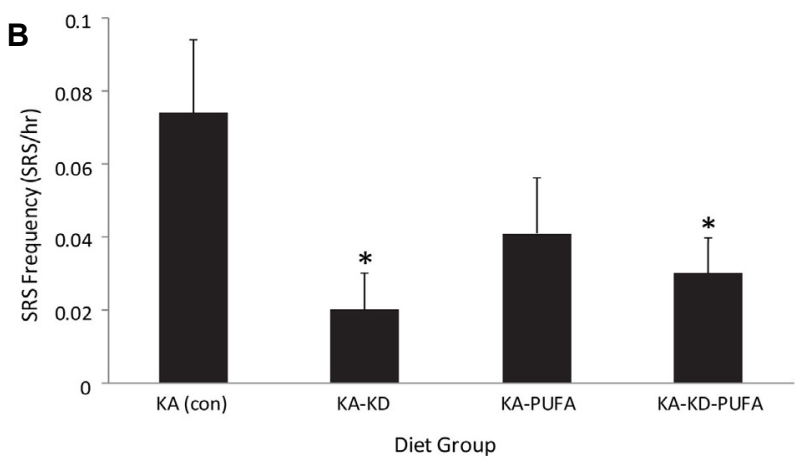

Figure 2. Spontaneous recurrent seizure (SRS) features following KA-induced status epilepticus as a function of dietary treatment. (A) Rats experiencing at least 1 or 5 SRS. Significantly fewer rats receiving the KD (KA-KD, KA-KD-PUFA) experienced at least 1 SRS compared to rats that did not receive the $K D$ (KA-con, KA-PUFA) $(\chi 2=6.465)$. Significantly fewer rats receiving the $K D(K A-K D, K A-K D-P U F A)$ experienced at least 5 SRS compared to rats that did not receive the KD (KA-con, KA-PUFA) $(\chi 2=4.876)$. (B) SRS frequency per hour among diet groups. SRS frequency was significantly less in rats receiving the KD (KA-KD, KA-KD-PUFA) compared to compared to rats that did not receive the KD (KA-con, KA-PUFA) $(p=0.038)$. (C) SRS per rat among diet groups. SRS per rat was significantly less in rats receiving the KD (KA-KD, KA-KD-PUFA) compared to compared to rats that did not receive the $K D(K A-c o n, K A-P U F A)(p=0.040)$. In all panels, data reported as mean \pm S.E.M. Asterisks $\left(^{*}\right)$ indicate significant differences compared to KA (con) groups. KA, kainic acid; PUFA, polyunsaturated fatty acid; KD, ketogenic diet, con, control.

neous seizures in the KA model in juvenile rats, supporting previous work. ${ }^{32}$ The PUFA diet alone did not result in greater protection against spontaneous seizure occurrence compared to controls in this model and the KD supplemented with PUFAs did not prevent seizures more than the straight KD.

PUFAs reportedly offer neuroprotection in several human diseases including Alzheimer disease, multiple sclerosis, learning disabilities, and attention deficit hyperactivity disorder, though the mechanism is unknown and rigorous clinical trials are lacking. ${ }^{39-41}$ PUFAs are also necessary for normal brain development. ${ }^{8,42}$ Given the observations that PUFAs reduce neuronal excitability, ${ }^{43}$ it is reasonable to hypothesize that PUFAs, especially those of the $n-3$ class, could have antiepileptic actions (decrease seizure occurrence in epileptic sub- 
jects) or even antiepileptogenic effects (prevent the development of epilepsy).

Preliminary clinical studies have evaluated a possible role of PUFAs in seizure control, with variable results - case reports found some benefitt ${ }^{23-25}$ while small clinical trials did not. ${ }^{26-29}$ An uncontrolled, unblinded clinical trial of only 5 patients reported reduced seizure frequency in an institutionalized population that received a PUFA-enhanced diet in the form of a fish oil bread spread..$^{23}$ In a case report, a 7-year-old boy with Lennox-Gastaut syndrome and a respiratory chain complex I deficiency benefitted from a PUFA-enriched Atkins diet with complete seizure control, EEG normalization, and cognitive improvement. ${ }^{24}$ In a dog with idiopathic epilepsy, dietary supplementation with $2 \mathrm{~g} /$ day of fish oil over several months brought seizures under control. ${ }^{25}$ However, not all clinical studies have shown a positive effect of PUFAs on seizures. A randomized, 12-week double blind study of PUFA supplementation versus placebo diet in 57 people with intractable epilepsy showed only a transient improvement in seizure control that was evident at 6 weeks of treatment but not thereafter. ${ }^{26}$ Another randomized study of PUFAs (EPA, DHA) versus placebo in 21 adult patients with uncontrolled epilepsy similarly reported no seizure improvement, ${ }^{27}$ nor did another 12-week double-blind crossover trial. ${ }^{28}$ In 25 children with intractable epilepsy placed on diets with fatty acid supplementation, there was no correlation between PUFA levels and seizure response. ${ }^{29}$

Animal studies of the effects of PUFAs on seizure control have also yielded variable results. Systemic administration of PUFAs raises the seizure threshold in some acute models, such as tail vein infusion of pentylenetretrazole, ${ }^{18}$ cortical stimulation, ${ }^{19}$ and 3-mercaptoproprionic acid-induced amygdala seizures. ${ }^{20}$ In other studies, a 1-month trial of dietary DHA or EPA did not produce an anticonvulsant effect ${ }^{21,22}$ and it has been suggested that a minimum of 3 months of dietary treatment is necessary to observe an anticonvulsant effect. ${ }^{44}$ Using the PTZ threshold test in rats, an intraperitoneal mixture of $n-3$ PUFAs was associated with elevated seizure thresholds, ${ }^{45}$ a result that could not be replicated. ${ }^{46}$ Rats fed a mixture of $n$-3 PUFAs (linoleic acid and $\alpha$-linolenic acid) exhibited an increase in PTZ seizure threshold, despite the lack of alteration of brain phospholipids. ${ }^{47}$ In contrast, mice fed DHA or EPA for 4 weeks had elevated fatty acid brain levels but did not have altered seizure thresholds in response to PTZ, flurothyl, or 6-Hz corneal stimulation or any change in status epilepticus severity in response to $\mathrm{KA}^{21}$

Therefore, variability in experimental designs and results does not allow a definitive conclusion regarding PUFA effects, at least in acute models. The current study represents one of the only studies of PUFAs using a chronic epilepsy model and one of the few using immature rats. Although we did not observe an added benefit of PUFAs, the finding that rats treated with a KD had significantly fewer spontaneous seizures than control-fed rats supports previous studies. ${ }^{32}$ The legitimate question arises as to whether body weight differences af-

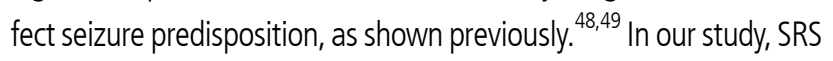
began about 1 month after KA and occurred in all treatment groups throughout the 3 months of diet treatment, irrespective of differences in body weights between groups; therefore, body weight differences cannot fully account for SRS differences in our study. In one study that used an isocaloric feeding protocol, there was no body weight difference or effect of diet treatment on the PTZ-seizure threshold at one month comparing control-, KD-, and linolenic acid-fed rats. ${ }^{47}$

Some of the discrepancies in results, at least in animal studies, may relate to the duration of treatment. Using the amygdala focal cortical stimulation model, it was recently shown that a minimum of 3 months was necessary for dietary PUFAs to show an anticonvulsant effect.44 These authors also commented that too low a dose of PUFAs may account for their failure to find an anticonvulsant action, an observation that may have implications for human trials.

We acknowledge the limitations of this study. Brain or serum levels of PUFAs were not measured. Spontaneous seizures were assessed visually, over limited time epochs, which could have led to an underestimation of seizure frequency. EEG or video EEG was not performed. Rats could have been experiencing electrographic seizures or class I-II seizures, which are difficult to detect visually. Doses of PUFAs were not varied. Nevertheless, our robust observation of fewer spontaneous seizures in both diet groups receiving the KD provides further evidence for the effectiveness of this diet in epilepsy and is consistent with the lack of effectiveness of PUFA supplementation in clinical epilepsy trials. It is possible that more refined PUFA protocols, perhaps using supplements with specific chain lengths or fat types, higher PUFA doses, or treatment for longer durations, might yield clinical benefit and emphasizes the need for further investigations in this area. ${ }^{44}$ For example, in rat hippocampal slices induced to generate epileptiform bursts in area CA1 with pentylenetetrazol or elevated extracellular potassium, perfusion of specific medium-chain triglycerides nonanoic acid or decanoic acid, but not octanoic acid (these are all straight-chain fatty acids) reversibly reduced epileptiform bursts..$^{50}$ Modification of octanoic acid by introduction of a side chain on carbon 4 (4-methyloctanoic acid) also reduced epi- 
leptiform bursts significantly, and using an in vivo perforant path stimulation model, this compound terminated status epilepticus. ${ }^{50}$ Therefore, specific chemical modifications of PUFAs could hold considerable promise for the control of seizures and epilepsy. As such, the present findings provide pilot data for further studies.

\section{Acknowledgements}

We are grateful to Dr. Stéphane Auvin and Dr. Ameer Y. Taha for helpful comments on the manuscript. Jennifer Broihahn provided superb assistance with figure preparation. This study was funded in part by a generous gift from the Mathias Koch Memorial Fund.

\section{Conflict of Interest}

The authors declare no conflicts of interest.

\section{References}

1. Kossoff EH, Zupec-Kania BA, Amark PE, et al. Optimal clinical management of children receiving the ketogenic diet: recommendations of the International Ketogenic Diet Study Group. Epilepsia 2009;50:304-17.

2. Gilbert DL, Pyzik PL, Freeman JM. The ketogenic diet: seizure control correlates better with serum beta-hydroxybutyrate than with urine ketones. J Child Neurol 2000;15:787-90.

3. Bough KJ, Yao SG, Eagles DA. Higher ketogenic diet ratios confer protection from seizures without neurotoxicity. Epilepsy Res 2000;38:15-25.

4. Samoilova M, Weisspapir M, Abdelmalik P, Velumian AA, Carlen PL. Chronic in vitro ketosis is neuroprotective but not anti-convulsant. $J$ Neurochem 2010;113:826-35.

5. Likhodii SS, Musa K, Mendonca A, Dell C, Burnham WB, Cunnane SC. Dietary fat, ketosis, and seizure resistance in rats on the ketogenic diet. Epilepsia 2000;41:1400-10.

6. Dell CA, Likhodii SS, Musa K, Ryan MA, Burnham WB, Cunnane SC. Lipid and fatty acid profiles in rats consuming different high-fat ketogenic diets. Lipids 2001;36:373-8.

7. Nylen K, Likhodii SS, Abdelmalik PA, Clarke J, Burnham WM. A comparison of the ability of a 4:1 ketogenic diet and a 6.3:1 ketogenic diet to elevate seizure thresholds in adult and young rats. Epilepsia 2005;46:1198-204.

8. Innis SM. The role of dietary n-6 and n-3 fatty acids in the developing brain. Dev Neurosci 2000;22:474-80.

9. Cunnane SC, Musa K, Ryan MA, Whiting S, Fraser DD. Potential role of polyunsaturates in seizure protection achieved with the ketogenic diet. Prostaglandins Leukot Essent Fatty Aci 2002;67:131-5.

10. Vreugdenhil M, Bruehl C, Voskuyl RA, Kang JX, Leaf A, Wadman WJ.
Polyunsaturated fatty acids modulate sodium and calcium currents in CA1 neurons. Proc Natl Acad Sci U S A 1996;93:12559-63.

11. Boland LM, Drzewiecki MM. Polyunsaturated fatty acid modulation of voltage-gated ion channels. Cell Biochem Biophys 2008;52:59-84.

12. $X u X P$, Erichsen $D$, Börjesson $S I$, Dahlin $M$, Amark $P$, Elinder $F$. Polyunsaturated fatty acids and cerebrospinal fluid from children on the ketogenic diet open a voltage-gated $\mathrm{K}$ channel: a putative mechanism of antiseizure action. Epilepsy Res 2008;80:57-66.

13. De Almeida $A C$, dos Santos $H L$, Rodrigues $A M$, et al. Non-synaptic mechanisms that could be responsible for potential antiepileptic effects of omega-3 fatty acids. Epilepsy Behav 2012;25:138-40.

14. Fraser DD, Whiting S, Andrew RD, Macdonald EA, Musa-Veloso K, Cunnane SC. Elevated polyunsaturated fatty acids in blood serum obtained from children on the ketogenic diet. Neurology 2003;60:1026-9.

15. Taha AY, Burnham WM, Auvin S. Polyunsaturated fatty acids and epilepsy. Epilepsia 2010;51:1348-58.

16. Musto $A E$, Gjorstrup $P$, Bazan NG. The omega-3 fatty acid-derived neuroprotectin D1 limits hippocampal hyperexcitability and seizure susceptibility in kindling epileptogenesis. Epilepsia 2011;52:1601-8.

17. Auvin S. Fatty acid oxidation and epilepsy. Epilepsy Res 2012;100:224-8.

18. Taha AY, Filo E, Ma DW, Mclntyre Burnham W. Dose-dependent anticonvulsant effects of linoleic and alpha-linolenic polyunsaturated fatty acids on pentylenetetrazol induced seizures in rats. Epilepsia 2009;50: 72-82.

19. Voskuyl RA, Vreugdenhil M, Kang JX, Leaf A. Anticonvulsant effect of polyunsaturated fatty acids in rats, using the cortical stimulation model. Eur J Pharmacol 1998;341:145-52.

20. Flores-Mancilla LE, Hernández-González M, Guevara MA, Benavides-Haro DE, Martínez-Arteaga P. Long-term fish oil supplementation attenuates seizure activity in the amygdala induced by 3-mercaptopropionic acid in adult male rats. Epilepsy Behav 2014;33:126-34.

21. Willis S, Samala R, Rosenberger TA, Borges K. Eicosapentaenoic and docosahexaenoic acids are not anticonvulsant or neuroprotective in acute mouse seizure models. Epilepsia 2009;50:138-42.

22. Curatolo $N$, Lecointe $C$, Bordet $R$, et al. Oral administration of docosahexaenoic acid/eicosapentaeinoic acids is not anticonvulsant in rats: implications for translational research. Pediatr Res 2011;70:584-8.

23. Schlanger $S$, Shinitzky M, Yam D. Diet enriched with omega-3 fatty acids alleviates convulsion symptoms in epilepsy patients. Epilepsia 2002:43:103-4.

24. Yoon JR, Lee EJ, Kim HD, Lee JH, Kang HC. Polyunsaturated fatty acid-enriched diet therapy for a child with epilepsy. Brain Dev 2014;36:163-6.

25. Scorza FA, Cavalheiro EA, Arida RM, et al. Positive impact of omega-3 fatty acid supplementation in a dog with drug-resistant epilepsy: a case study. Epilepsy Behav 2009;15:527-8.

26. Yuen AW, Sander JW, Fluegel D, et al. Omega-3 fatty acid supplementation in patients with chronic epilepsy: a randomized trial. Epilepsy Behav 2005;7:253-8. 
27. Bromfield E, Dworetzky B, Hurwitz $\mathrm{S}$, et al. A randomized trial of polyunsaturated fatty acids for refractory epilepsy. Epilepsy Behav 2008; 12:187-90.

28. DeGiorgio CM, Miller P, Meymandi S, Gornbein JA. n-3 fatty acids (fish oil) for epilepsy, cardiac risk factors, and risk of SUDEP: clues from a pilot, double-blind, exploratory study. Epilepsy Behav 2008;13:681-4.

29. Dahlin M, Hjelte L, Nilsson S, Amark P. Plasma phospholipid fatty acids are influenced by a ketogenic diet enriched with n-3 fatty acids in children with epilepsy. Epilepsy Res 2007;73:199-207.

30. Ben-Ari Y. Limbic seizure and brain damage produced by kainic acid: mechanisms and relevance to human temporal lobe epilepsy. Neuroscience 1985;14:375-403.

31. Stafstrom CE, Thompson JL, Holmes GL. Kainic acid seizures in the developing brain: status epilepticus and spontaneous recurrent seizures. Brain Res Dev Brain Res 1992;65:227-36.

32. Su SW, Cilio MR, Sogawa Y, Silveira DC, Holmes GL, Stafstrom CE. Timing of ketogenic diet initiation in an experimental epilepsy model. Brain Res Dev Brain Res 2000;125:131-8.

33. Rogers AE. Nutrition. In: Baker HJ, Lindsey JR, Weisbroth SH, eds. The Laboratory Rat. Orlando: Academic Press, 1979: 123-52.

34. Bough KJ, Schwartzkroin PA, Rho JM. Calorie restriction and ketogenic diet diminish neuronal excitability in rat dentate gyrus in vivo. Epilepsia 2003;44:752-60.

35. Racine RJ. Modification of seizure activity by electrical stimulation: II. Motor seizure. Electroencephalogr Clin Neurophysiol 1972;32:281-94.

36. Thio LL, Erbayat-Altay E, Rensing N, Yamada KA. Leptin contributes to slower weight gain in juvenile rodents on a ketogenic diet. Pediatr Res 2006:60:413-7.

37. Peres RC, Nogueira DB, de Paula Guimarães G, da Costa EL, Ribeiro DA. Implications of ketogenic diet on weight gain, motor activity and cicatrization in Wistar rats. Toxicol Mech Methods 2013;23:144-9.

38. Wachira JK, Larson MK, Harri WS. n-3 Fatty acids affect haemostasis but do not increase the risk of bleeding: clinical observations and mechanistic insights. Br J Nutr 2014;111:1652-62.

39. Calon F. Omega-3 polyunsaturated fatty acids in Alzheimer's disease: key questions and partial answers. Curr Alzheimer Res 2011;8:470-8.

40. von Geldern G, Mowry EM. The influence of nutritional factors on the prognosis of multiple sclerosis. Nat Rev Neurol 2012;8:678-89.

41. Gillies D, Sinn JKH, Lad SS, Leach MJ, Ross MJ. Polyunsaturated fatty acids (PUFA) for attention deficit hyperactivity disorder (ADHD) in children and adolescents. Cochrane Database Syst Rev 2012;7:CD007986.

42. Salvati S, Attorri L, Avellino C, Di Biase A, Sanchez M. Diet, lipids and brain development. Dev Neurosci 2000;22:481-7.

43. Young C, Gean PW, Chiou LC, Shen YZ. Docosahexaenoic acid inhibits synaptic transmission and epileptiform activity in the rat hippocampus. Synapse 2000;37:90-4.

44. Taha AY, Trepanier MO, Ciobanu FA, et al. A minimum of 3 months of dietary fish oil supplementation is required to raise amygdaloid afterdischarge seizure thresholds in rats-implications for treating complex partial seizures. Epilepsy Behav 2013;27:49-58.

45. Yehuda S, Carasso RL, Mostofsky DI. Essential fatty acid preparation (SR-3) raises the seizure threshold in rats. Eur I Pharmacol 1994;254:193-8.

46. Taha AY, Baghiu BM, Lui R, Nylen K, Ma DW, Burnham WM. Lack of benefit of linoleic and alpha-linolenic polyunsaturated fatty acids on seizure latency, duration, severity or incidence in rats. Epilepsy Res 2006;71:40-6.

47. Porta N, Bourgois B, Galabert C, et al. Anticonvulsant effects of linolenic acid are unrelated to brain phospholipid cell membrane compositions. Epilepsia 2009;50:65-71.

48. Bough KJ, Valiyil R, Han FT, Eagles DA. Seizure resistance is dependent upon age and calorie restriction in rats fed a ketogenic diet. Epilepsy Res 1999;35:21-8.

49. Mantis JG, Centeno NA, Todorova MT, McGowan R, Seyfried TN. Management of multifactorial idiopathic epilepsy in EL mice with caloric restriction and the ketogenic diet: role of glucose and ketone bodies. Nutr Metab (Lond) 2004;1:11.

50. Chang P, Terbach N, Plant N, Chen PE, Walker MC, Williams RS. Seizure control by ketogenic diet-associated medium chain fatty acids. Neuropharmacology 2013;69:105-14. 